\title{
Irradiated benzene ice provides clues to meteoritic organic chemistry
}

\author{
Michael P. Callahan ${ }^{\mathrm{a}, \mathrm{b}, *}$, Perry A. Gerakines ${ }^{\mathrm{a}, \mathrm{b}}$, Mildred G. Martin ${ }^{\mathrm{a}, \mathrm{b}, \mathrm{c}}$, Zan Peeters ${ }^{\mathrm{d}}$, Reggie L. Hudson ${ }^{\mathrm{a}, \mathrm{b}}$ \\ a Solar System Exploration Division, National Aeronautics and Space Administration Goddard Space Flight Center, Greenbelt, MD 20771, USA \\ ${ }^{\mathrm{b}}$ Goddard Center for Astrobiology, National Aeronautics and Space Administration Goddard Space Flight Center, Greenbelt, MD 20771, USA \\ ${ }^{\mathrm{c}}$ Catholic University of America, Washington, DC 20064, USA \\ ${ }^{\mathrm{d}}$ Department of Terrestrial Magnetism, Carnegie Institution of Washington, Washington, DC 20015, USA
}

\section{A R T I C L E I N F O}

\section{Article history:}

Received 21 December 2012

Revised 24 July 2013

Accepted 27 July 2013

Available online 13 August 2013

\section{Keywords:}

Astrobiology

Cosmic rays

Cosmochemistry

Experimental techniques

Ices, IR spectroscopy

\begin{abstract}
A B S T R A C T
Aromatic hydrocarbons account for a significant portion of the organic matter in carbonaceous chondrite meteorites, as a component of both the low molecular weight, solvent-extractable compounds and the insoluble organic macromolecular material. Previous work has suggested that the aromatic compounds in carbonaceous chondrites may have originated in the radiation-processed icy mantles of interstellar dust grains. Here we report new studies of the organic residue made from benzene irradiated at $19 \mathrm{~K}$ by $0.8 \mathrm{MeV}$ protons. Polyphenyls with up to four rings were unambiguously identified in the residue by gas chromatography-mass spectrometry. Atmospheric pressure photoionization Fourier transform mass spectrometry was used to determine molecular composition, and accurate mass measurements suggested the presence of polyphenyls, partially hydrogenated polyphenyls, and other complex aromatic compounds. The profile of low molecular weight compounds in the residue compared well with extracts from the Murchison and Orgueil meteorites. These results are consistent with the possibility that solid phase radiation chemistry of benzene produced some of the complex aromatics found in meteorites.
\end{abstract}

Published by Elsevier Inc.

\section{Introduction}

Meteorites preserve a record of the chemistry that occurred in the early Solar System before the planets fully accreted. Carbonaceous chondrites are among the most primitive of meteorites, but they are also the most chemically complex (Schmitt-Kopplin et al., 2010). They contain a plethora of organic molecules, including many that are essential in contemporary biology (Callahan et al., 2011; Cooper et al., 2001, 2011; Cronin and Pizzarello, 1997; Kvenvolden et al., 1970; Meierhenrich et al., 2004; Yuen and Kvenvolden, 1973), and their delivery to the early Earth could have been an important source of prebiotic compounds for the emergence of life (Chyba and Sagan, 1992; Oró, 1961).

Aromatic hydrocarbons account for a significant portion $(\sim 5 \%)$ of the free (solvent-extracted) organic matter in carbonaceous chondrites (Mullie and Reisse, 1987), and are related to the insoluble macromolecular material, which composes $70-99 \%$ of the organic matter in these same meteorites (Sephton et al., 1998). The aromatics in the Murchison meteorite are enriched in deuterium (Kerridge et al., 1987), which suggests their low temperature chemical synthesis. Sephton and Gilmour used gas chromatography-isotope ratio mass spectrometry to measure $\delta^{13} \mathrm{C}$ values for

\footnotetext{
* Corresponding author at: Solar System Exploration Division, National Aeronautics and Space Administration Goddard Space Flight Center, Greenbelt, MD 20771, USA. Fax: +1 (301) 2861683.

E-mail address: michael.p.callahan@nasa.gov (M.P. Callahan).
}

individual free aromatic compounds in the Cold Bokkeveld and Murchison meteorites, and they concluded that these compounds were synthesized from radiation induced reactions, most likely in the icy organic mantles of interstellar grains (Sephton and Gilmour, 2000; Sephton et al., 2000). Furthermore, theoretical modeling has shown that warming, in addition to irradiation, would be natural consequences of the dynamical evolution of ice grains in the solar nebula, which would facilitate the production of complex organics (Ciesla and Sandford, 2012).

Among all aromatic molecules, benzene $\left(\mathrm{C}_{6} \mathrm{H}_{6}\right)$ is the simplest neutral cyclic hydrocarbon (Fig. 1), and it has been identified in a diverse range of extraterrestrial materials and environments. For example, benzene has been found in carbonaceous chondrites (Sephton, 2002), in the circumstellar medium of the protoplanetary nebula CRL618 (Cernicharo et al., 2001), and also in Titan's atmosphere (Coustenis et al., 2003; Flasar et al., 2005; Waite et al., 2005). Ionizing radiation in circumstellar and interstellar environments will alter benzene and all other organics, and it has been postulated that Titan's atmospheric benzene could be processed by Saturn's magnetosphere to form higher molecular weight products (Delitsky and McKay, 2010). Moreover, benzene has been suggested as an intermediate in the formation of polycyclic aromatic hydrocarbons (PAHs, Fig. 1) and other organics (Frenklach and Feigelson, 1989).

Given such a rich set of applications involving benzene, it is surprising that so little work has been done on the radiation chemistry of solid $\mathrm{C}_{6} \mathrm{H}_{6}$. Strazzulla and Baratta (1991) showed that more 


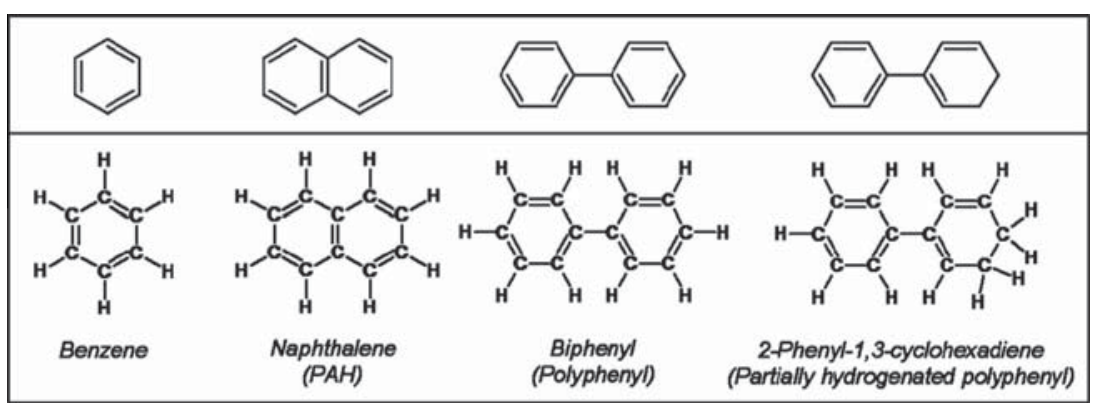

Fig. 1. The general structures of benzene, polycyclic aromatic hydrocarbons or PAHs (such as naphthalene, $\mathrm{C}_{10} \mathrm{H}_{8}$ ), polyphenyls (such as biphenyl, $\mathrm{C}_{12} \mathrm{H}_{10}$ ), and partially

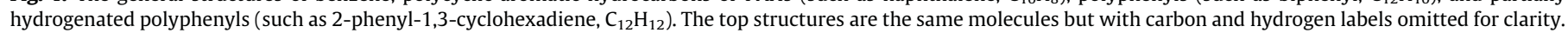

complex molecules were formed after frozen benzene was irradiated with a 3-keV $\mathrm{He}^{+}$beam; however, their analysis of the postirradiation organic residue was limited to infrared (IR) spectroscopy. Later experiments in our laboratory by Ruiterkamp et al. (2005) determined the stability of solid, matrix-isolated, and $\mathrm{H}_{2} \mathrm{O}$ ice-embedded benzene exposed to $0.8-\mathrm{MeV} \mathrm{H}^{+}$, and showed that $\mathrm{C}_{6} \mathrm{H}_{6}$ could survive in interstellar dense clouds on the surfaces of icy grains and in circumstellar envelopes if sufficient shielding existed. Still, the organic residue after irradiation was not examined in detail.

Because of this contrast between multiple applications of benzene chemistry and the paucity of associated laboratory work, we recently have investigated the radiation processing of $\mathrm{C}_{6} \mathrm{H}_{6}$ at low temperatures $(<20 \mathrm{~K})$. Here, we report a significant extension of our previous studies (Ruiterkamp et al., 2005), now emphasizing the composition of the refractory residue recovered from irradiated frozen benzene. We have applied the highly sensitive analytical techniques of gas chromatography-mass spectrometry (GC-MS) and atmospheric pressure photoionization Fourier transform mass spectrometry (APPI-FTMS) for molecular characterization of this residue. We also have analyzed solvent extracts from three different meteorites for comparison to possible radiation induced aromatic chemistry on meteorite parent bodies. While the present paper is restricted to connections of our experiments to meteorites, our results may also be relevant to Titan and interstellar chemistry. We note that benzene serves as a model compound for aromatic chemistry as we expect that the products and processes described here are relevant for other aromatic molecules.

\section{Experimental methods}

\subsection{Radiation experiments}

Proton irradiation experiments were performed in the Cosmic Ice Laboratory at the NASA Goddard Space Flight Center. The system used consisted of a stainless steel high vacuum chamber containing a polished aluminum substrate attached to the cold finger of a closed-cycle helium cryostat (ARS model DE-204). This rotatable substrate can face a deposition tube, a beam of $0.8-\mathrm{MeV}$ protons from a Van de Graaff accelerator, or an FT-IR spectrometer (Hudson and Moore, 2003, 2004). By leaking benzene vapor into the vacuum chamber, a $\mathrm{C}_{6} \mathrm{H}_{6}$ ice (thickness $\approx 11 \mu \mathrm{m}$ ) was grown on the substrate, which was held at $19 \mathrm{~K}$. Contaminants (mainly $\mathrm{H}_{2} \mathrm{O}$ ) made up no more than $1 \%$ of the final ice, based on our estimates of the background gases present and the rate at which the sample was grown.

After deposition, the benzene ice was exposed to a $0.8-\mathrm{MeV}$ proton beam with a current of $150 \mathrm{nA}$ until a fluence of $3 \times 10^{15}$ protons $\mathrm{cm}^{-2}$ was achieved. The average energy dose (energy absorbed) was $119 \mathrm{eV}$ per initial benzene molecule deposited, assuming a proton stopping power for solid $\mathrm{C}_{6} \mathrm{H}_{6}$ of $307.1 \mathrm{MeV} \mathrm{cm}^{2} \mathrm{~g}^{-1}$ proton ${ }^{-1}$. Two experiments at low temperature $(\sim 20 \mathrm{~K})$ and irradiation were conducted as well as another experiment at higher temperature $(150 \mathrm{~K})$. For descriptions of the IR spectra of proton-irradiated frozen benzene, see Strazzulla and Baratta (1991) and Ruiterkamp et al. (2005).

\subsection{Gas chromatography-mass spectrometry measurements}

After irradiation, the benzene ice sample was kept under vacuum and allowed to slowly warm to room temperature overnight, permitting any volatile products and unreacted benzene to be pumped away. The remaining material, which we refer to as a refractory organic residue, was removed from the substrate by washing with $1 \mathrm{~mL}$ of HPLC grade (99.9\%) dichloromethane $\left(\mathrm{CH}_{2-}\right.$ $\mathrm{Cl}_{2}$ ). The sample extract was dried under vacuum then re-dissolved in $100 \mu \mathrm{L}$ of dichloromethane to concentrate the extract.

A Finnigan TraceGC ultra gas chromatograph was used for this study, equipped with a Restek Rxi-5 ms column $(30 \mathrm{~m}, 0.25 \mathrm{~mm}$ ID, $0.5 \mu \mathrm{m}$ film thickness) and coupled to a Finnigan TraceDSQ quadrupole mass spectrometer. A sample injection volume of $1 \mu \mathrm{L}$, injector temperature of $275^{\circ} \mathrm{C}$, transfer line temperature of $280^{\circ} \mathrm{C}$, and a flow of $1.2 \mathrm{~mL} \mathrm{~min}^{-1}$ helium were used. The column temperature was held at $75^{\circ} \mathrm{C}$ for $30 \mathrm{~s}$, increased to $245^{\circ} \mathrm{C}$ at $25^{\circ} \mathrm{C} \mathrm{min}{ }^{-1}$, increased again to $330^{\circ} \mathrm{C}$ at $4{ }^{\circ} \mathrm{C} \mathrm{min}{ }^{-1}$, and then held at $330^{\circ} \mathrm{C}$ for $15 \mathrm{~min}$. The ionization source used $70 \mathrm{eV}$ electron energies and was kept at $200{ }^{\circ} \mathrm{C}$. Mass spectra were recorded from $m / z=50-650 \mathrm{Da}$, and a solvent delay time of $4 \mathrm{~min}$ was employed. Compound identifications were made by comparing chromatographic retention times and electron ionization mass spectra with commercial reference standards obtained from Sigma Aldrich. In addition, a mass spectral library (Wiley Registry 8th Edition/NIST 2005) was searched for possible compound identification.

\subsection{Atmospheric pressure photoionization-mass spectrometry (APPI- MS) measurements}

APPI-MS analyses were conducted with an Accela 1250 ultrahigh precision liquid chromatography pump and autosampler (Thermo Scientific) coupled to a LTQ Orbitrap XL hybrid mass spectrometer (Thermo Scientific) fitted with an atmospheric pressure photoionization (APPI) source. In APPI, a sample solution is vaporized and then ionized by photons. The APPI source uses a krypton lamp that emits photons at 10.0 and $10.6 \mathrm{eV}$, and was chosen because it can ionize, with minimal fragmentation, both polar and nonpolar molecules (Hanold et al., 2004; Robb et al., 2000). The analyte molecule can be ionized directly (direct photoionization) or ionized indirectly with the help of solvent molecules or inclusion of a dopant. In our analyses, protonation of neutral analytes likely occurred via photoionization-induced chemical ionization 
where photoionization of the methanol mobile phase produced protonated methanol clusters which then led to chemical ionization of an analyte (Short et al., 2007).

For the flow-injection analyses, $10 \mu \mathrm{L}$ of sample were injected at a flow rate of $50 \mu \mathrm{L} \mathrm{min}^{-1}$ using methanol as the mobile phase. For APPI, the vaporizer temperature was $375^{\circ} \mathrm{C}$, the sheath gas flow was 25 (arbitrary units), the auxiliary gas flow was 5 (arbitrary units), and the sweep gas flow was 1 (arbitrary units). The sheath, auxiliary, and sweep gases were all nitrogen, and these settings assisted in the vaporization of the sample solution. The APPI lamp was positioned 1-2 $\mathrm{mm}$ from the orifice of the ion transfer capillary. The ion transfer capillary voltage and ion transfer capillary temperature were $18 \mathrm{~V}$ and $200^{\circ} \mathrm{C}$, respectively. The tube lens was set at $45 \mathrm{~V}$. The mass spectrometer was operated in positive ion mode and the mass resolution was set to 100,000 (full-width at half-maximum for $m / z=400$ ). A high resolution mass spectrum captures a "molecular snapshot" of all the organic compounds in the sample attainable from dichloromethane extraction and APPI. In addition, the instrument's high mass accuracy enables the assignment of elemental composition to mass peaks, which can be used for molecular characterization.

The mass spectrometer was externally calibrated using a mixture of caffeine, MRFA (L-methionyl-arginyl-phenylalanyl-alanine acetate hydrate) peptide, and Ultramark 1621 in an acetonitrilemethanol-water solution containing $1 \%$ acetic acid. Electrospray ionization was used because this method of ionization does not affect the calibration. After external mass calibration, the electrospray ionization probe was replaced by the APPI probe and lamp. A background compound $\left(m / z=87.08044,\left[\mathrm{C}_{5} \mathrm{H}_{10} \mathrm{O}+\mathrm{H}\right]^{+}\right)$was used for internal calibration, which resulted in a typical relative mass error under 2 parts-per-million (ppm). For the benzene residue, each molecular component detected was assumed to be composed of only the elements $\mathrm{C}, \mathrm{H}$, and $\mathrm{O}$, where residual water in the irradiation setup could be a source of oxygen contamination.

\subsection{Meteorite extractions}

Sample handling tools, ceramics, and glassware were rinsed with ultrapure water $(18.2 \mathrm{M} \Omega \mathrm{cm},<5 \mathrm{ppb}$ total organic carbon from a Millipore Milli-Q Integral 10 system), then wrapped in aluminum foil, and then heated under air at $500{ }^{\circ} \mathrm{C}$ for $24 \mathrm{~h}$ to remove any organic contaminants. Meteorites were crushed to a powder using a porcelain mortar and pestle in a Class-100 laminar flow hood under HEPA-filtered positive pressure. The powdered meteorite samples (14.8 mg for Murchison meteorite, $11.6 \mathrm{mg}$ for Orgueil, and $12.6 \mathrm{mg}$ for Roberts Massif (RBT) 04262) were transferred to borosilicate centrifuge vials and $300 \mu \mathrm{L}$ dichloromethane was added to each before sealing with a PFTE-lined screw cap. The vials were put into a water bath-shaker maintained at $35^{\circ} \mathrm{C}$, and then agitated overnight in order to extract the soluble organic matter from the meteorite powder. Finally, the vials were centrifuged to isolate the meteorite extracts, which were transferred to autosampler vials and analyzed by APPI mass spectrometry.

\section{Results}

\subsection{Aromatic products in the residue from proton-irradiated benzene}

Fig. 2 shows the GC-MS total ion chromatogram of the dichloromethane extract of the room temperature benzene residue. The compounds corresponding to the most intense peaks in the chromatogram were identified as biphenyl $\left(\left(\mathrm{C}_{6} \mathrm{H}_{5}\right)_{2}\right.$, sometimes called diphenyl) followed in intensity by $o$-terphenyl, $m$-terphenyl, and p-terphenyl. Several quaterphenyls likely were present based on strong molecular ion peaks and late retention times (between 12 and $22 \mathrm{~min}$ ), although due to a lack of reference standards only 1,2,3-triphenylbenzene, and 1,3,5-triphenylbenzene could be identified. All of the molecules identified above belong to the class of compounds known as polyphenyls (Fig. 1), which are composed of benzene rings (phenyl groups) linked only by $\mathrm{C}-\mathrm{C}$ bonds.

The GC-MS chromatogram of Fig. 2 also contains numerous smaller peaks, which suggested that the benzene residue contained other complex organic compounds. Some of these peaks are consistent with substituted aromatic compounds, which may have resulted from ring opening of one of the aromatic rings in biphenyl; however no firm identifications can be made based on our mass spectral library. Larger polyphenyls (five rings and greater) were not detected, but may also be present because GC-MS analysis is often unable to detect organic compounds with low volatility, low thermal stability, and/or large size. Finally, we used our GC-MS method to search for the following PAHs in the benzene residue: naphthalene, anthracene, phenanthrene, pyrene,

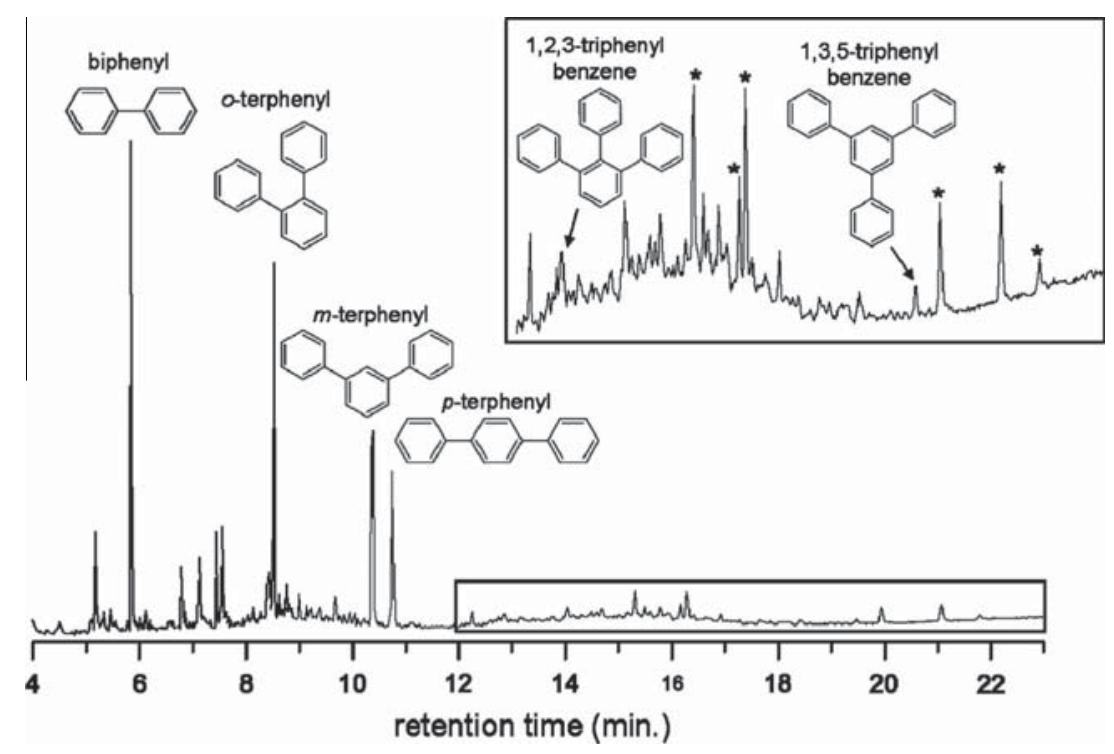

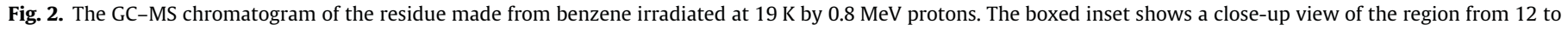
$23 \mathrm{~min}$. Peaks labeled with an asterisk are assigned to other quaterphenyl isomers based on their mass spectra. 
1,2-benzanthracene, perylene, benzo[g,h,i]perylene, and coronene. None of these PAHs were detected above our detection limits $(\sim 10 \mathrm{pmol})$. Some of the smaller peaks in the GC-MS chromatogram may be substituted PAHs, but definitive assignments were impossible using only our mass spectral library because substituted polyphenyls also have very similar mass spectra.

Fig. 3 shows the residue's APPI mass spectrum, which is complex with over a thousand peaks. The high mass accuracy of the Orbitrap analyzer, along with the utilization of the natural isotope pattern, enabled the assignment of elemental formulae for small molecules. It is worth noting that a single elemental formula could represent numerous structural isomers. The high resolution mass spectrometry techniques employed here may represent a more accurate picture of the sample's chemical diversity compared to the GC-MS results.

Mass envelopes with prominent peaks separated by 76 Da were observed, which indicated a phenyl group addition and hydrogen elimination. Elemental formulae from accurate mass measurements confirmed the presence of polyphenyls as protonated species from APPI. Proton irradiation of benzene also resulted in partially hydrogenated polyphenyls (Fig. 1) and other partially hydrogenated aromatic hydrocarbons based on the repeated addition of two hydrogen atoms observed in the mass spectrum (Fig. 3 and Table 1). Additionally, larger molecular weight compounds display a greater extent of hydrogenation because there are more opportunities to include partially hydrogenated aromatic rings into the structure as the polyphenyl chain increases in size. Hydrogen atom addition to aromatic rings has been reported as partially hydrogenated aromatic hydrocarbons were formed during the UV irradiation of PAHs in low temperature ices (Bernstein et al., 1999, 2002; Gudipati and Yang, 2012) and from the gamma radiolysis of liquid benzene (Gordon et al., 1958). Superhydrogenated derivatives of the PAH coronene through $\mathrm{H}$-atom addition reactions also have been demonstrated at high temperatures (Thrower et al., 2012). These modifications transform some of the aromatic rings into cyclic aliphatic hydrocarbon structures, which reduce the overall degree of aromaticity in the molecule and change their spectroscopic signature (e.g., a 3.4- $\mu \mathrm{m}$ band, due to the presence of aliphatic carbon, may appear next to the aromatic 3.3- $\mu \mathrm{m}$ band).

High molecular weight species $(m / z \sim 800 \mathrm{Da})$, which were inaccessible by our GC-MS methods, were observed in the APPI mass spectrum (Fig. 3). Assignments using accurate mass measurements are less certain for high molecular weight species because the list of potential elemental formulae becomes very large despite constant mass accuracy. However, if we assume that polyphenyl synthesis could extend to higher masses, we observe elemental formulae consistent with large polyphenyls containing up to ten rings, although for large compounds containing eight rings or more, all products were partially hydrogenated. Our assumption is verified via carbon-normalized double bond equivalents (DBE/ C) analysis, previously used in ultrahigh resolution mass spectrometry studies (Hockaday et al., 2006), to provide additional insight on the aromaticity of a given molecule. A DBE/C $\geqslant 0.7$ suggests fused aromatic ring structures (such as PAHs). For peaks with $\mathrm{S} /$ $\mathrm{N}>10$, only $3 \%$ of the mass peaks have a $\mathrm{DBE} / \mathrm{C} \geqslant 0.7$, which suggests the majority of the peaks are "polyphenyl-like" as opposed to "PAH-like". It is worth pointing out that there is a very weak peak in the APPI mass spectrum that corresponds to protonated naphthalene; however, additional information (e.g., chromatographic retention time, product ion spectrum) would be needed for unambiguous identification.

Finally, oxygenated species are present throughout the APPI mass spectrum (Fig. 3). Despite the fact that proton irradiation was performed under vacuum, some residual water was likely trapped within the sample and could have reacted with benzene to form oxygenated species. Some oxygenation may also have occurred during exposure to the atmosphere after warm-up, extraction, or during analysis.

\subsection{Meteorite results and comparison to the benzene residue}

The high-resolution mass spectrum of the benzene residue (Fig. 3) shows a large number of mass peaks. The peak intensity reaches a maximum for the 4-ring group; however, this group may not represent the most abundant compounds because peak intensity depends on multiple factors (such as ionization efficiency). Thus, APPI mass spectral data should be considered qualitative only. This point is further illustrated when this data is compared with GC-MS data of the benzene residue, which shows that peak intensity decreases as molecular size increases (i.e., 2ring > 3-ring > 4-ring, etc.), which likely resembles its true distribution. While assigning every peak in such a spectrum is nearly impossible, in some cases such complexity can be beneficial. Along those lines, we selected a set of 69 of the strongest peaks from $\mathrm{m} /$ $z=120-320$ to use as a "fingerprint" of the radiation chemistry of frozen benzene. This set of intense peaks represented diagnostic hydrocarbon ions, and were selected because they most likely correspond to polyphenyls, partially hydrogenated polyphenyls, and other complex aromatic compounds. Table 1 lists the selected ions,

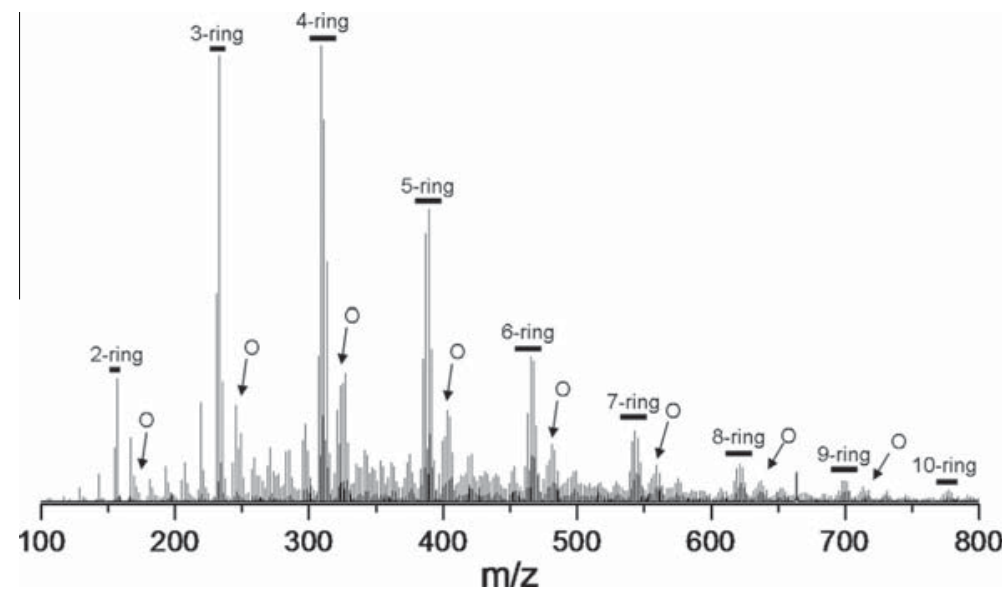

Fig. 3. APPI mass spectrum of the residue made from benzene irradiated at $19 \mathrm{~K}$ by $0.8 \mathrm{MeV}$ protons. Polyphenyls and partially hydrogenated polyphenyls up to 10 -ring compounds are indicated by the horizontal lines. Mass envelopes of oxygenated species also are present, and are marked by "O". 
Table 1

Benzene residue diagnostic ions in meteorites.

\begin{tabular}{|c|c|c|c|c|c|c|}
\hline Measured $m / z$ & Theoretical $\mathrm{m} / \mathrm{z}$ & Mass error (ppm) & Formula & Murchison (CM2) & Orgueil (CI) & RBT 04262 (SNC) \\
\hline 129.0699 & 129.0699 & -0.19 & $\mathrm{C}_{10} \mathrm{H}_{9}$ & + & + & - \\
\hline 131.0855 & 131.0855 & -0.22 & $\mathrm{C}_{10} \mathrm{H}_{11}$ & + & + & - \\
\hline 141.0700 & 141.0699 & 1.01 & $\mathrm{C}_{11} \mathrm{H}_{9}$ & + & + & - \\
\hline 143.0856 & 143.0855 & 0.45 & $\mathrm{C}_{11} \mathrm{H}_{11}$ & + & + & - \\
\hline 155.0854 & 155.0855 & -0.88 & $\mathrm{C}_{12} \mathrm{H}_{11}$ & + & + & - \\
\hline 157.1011 & 157.1012 & -0.54 & $\mathrm{C}_{12} \mathrm{H}_{13}$ & + & + & - \\
\hline 167.0854 & 167.0855 & -0.73 & $\mathrm{C}_{13} \mathrm{H}_{11}$ & + & + & - \\
\hline 169.1010 & 169.1012 & -1.17 & $\mathrm{C}_{13} \mathrm{H}_{13}$ & + & + & - \\
\hline 171.1167 & 171.1168 & -0.98 & $\mathrm{C}_{13} \mathrm{H}_{15}$ & + & + & + \\
\hline 179.0856 & 179.0855 & 0.61 & $\mathrm{C}_{14} \mathrm{H}_{11}$ & + & + & - \\
\hline 181.1011 & 181.1012 & -0.45 & $\mathrm{C}_{14} \mathrm{H}_{13}$ & + & + & - \\
\hline 183.1167 & 183.1168 & -0.79 & $\mathrm{C}_{14} \mathrm{H}_{15}$ & + & + & - \\
\hline 185.1323 & 185.1325 & -0.82 & $\mathrm{C}_{14} \mathrm{H}_{17}$ & + & + & - \\
\hline 191.0853 & 191.0855 & -0.99 & $\mathrm{C}_{15} \mathrm{H}_{11}$ & + & + & - \\
\hline 193.1011 & 193.1012 & -0.60 & $\mathrm{C}_{15} \mathrm{H}_{13}$ & + & + & - \\
\hline 195.1167 & 195.1168 & -0.53 & $\mathrm{C}_{15} \mathrm{H}_{15}$ & + & + & - \\
\hline 197.1324 & 197.1325 & -0.40 & $\mathrm{C}_{15} \mathrm{H}_{17}$ & + & + & - \\
\hline 199.1481 & 199.1481 & -0.37 & $\mathrm{C}_{15} \mathrm{H}_{19}$ & + & + & - \\
\hline 205.1011 & 205.1012 & -0.51 & $\mathrm{C}_{16} \mathrm{H}_{13}$ & + & + & - \\
\hline 207.1168 & 207.1168 & -0.10 & $\mathrm{C}_{16} \mathrm{H}_{15}$ & + & + & - \\
\hline 209.1323 & 209.1325 & -0.73 & $\mathrm{C}_{16} \mathrm{H}_{17}$ & + & + & - \\
\hline 211.1481 & 211.1481 & 0.09 & $\mathrm{C}_{16} \mathrm{H}_{19}$ & + & + & - \\
\hline 213.1638 & 213.1638 & 0.19 & $\mathrm{C}_{16} \mathrm{H}_{21}$ & + & + & - \\
\hline 217.1010 & 217.1012 & -0.92 & $\mathrm{C}_{17} \mathrm{H}_{13}$ & + & + & - \\
\hline 219.1168 & 219.1168 & -0.27 & $\mathrm{C}_{17} \mathrm{H}_{15}$ & + & + & - \\
\hline 221.1324 & 221.1325 & -0.47 & $\mathrm{C}_{17} \mathrm{H}_{17}$ & + & + & - \\
\hline 223.1480 & 223.1481 & -0.77 & $\mathrm{C}_{17} \mathrm{H}_{19}$ & + & + & - \\
\hline 225.1637 & 225.1638 & -0.21 & $\mathrm{C}_{17} \mathrm{H}_{21}$ & + & + & - \\
\hline 227.1795 & 227.1794 & 0.15 & $\mathrm{C}_{17} \mathrm{H}_{23}$ & + & + & - \\
\hline 229.1013 & 229.1012 & 0.35 & $\mathrm{C}_{18} \mathrm{H}_{13}$ & + & + & - \\
\hline 231.1167 & 231.1168 & -0.35 & $\mathrm{C}_{18} \mathrm{H}_{15}$ & + & + & - \\
\hline 233.1325 & 233.1325 & -0.09 & $\mathrm{C}_{18} \mathrm{H}_{17}$ & + & + & - \\
\hline 235.1481 & 235.1481 & -0.13 & $\mathrm{C}_{18} \mathrm{H}_{19}$ & + & + & - \\
\hline 237.1637 & 237.1638 & -0.25 & $\mathrm{C}_{18} \mathrm{H}_{21}$ & + & + & - \\
\hline 239.1795 & 239.1794 & 0.34 & $\mathrm{C}_{18} \mathrm{H}_{23}$ & + & + & - \\
\hline 243.1168 & 243.1168 & -0.26 & $\mathrm{C}_{19} \mathrm{H}_{15}$ & + & + & - \\
\hline 245.1325 & 245.1325 & 0.02 & $\mathrm{C}_{19} \mathrm{H}_{17}$ & + & + & - \\
\hline 247.1481 & 247.1481 & -0.21 & $\mathrm{C}_{19} \mathrm{H}_{19}$ & + & + & - \\
\hline 249.1637 & 249.1638 & -0.43 & $\mathrm{C}_{19} \mathrm{H}_{21}$ & + & + & - \\
\hline 251.1795 & 251.1794 & 0.29 & $\mathrm{C}_{19} \mathrm{H}_{23}$ & + & + & - \\
\hline 255.1167 & 255.1168 & -0.34 & $\mathrm{C}_{20} \mathrm{H}_{15}$ & - & + & - \\
\hline 257.1324 & 257.1325 & -0.41 & $\mathrm{C}_{20} \mathrm{H}_{17}$ & - & + & - \\
\hline 259.1481 & 259.1481 & -0.30 & $\mathrm{C}_{20} \mathrm{H}_{19}$ & + & + & - \\
\hline 261.1638 & 261.1638 & 0.20 & $\mathrm{C}_{20} \mathrm{H}_{21}$ & + & + & - \\
\hline 263.1793 & 263.1794 & -0.50 & $\mathrm{C}_{20} \mathrm{H}_{23}$ & + & + & - \\
\hline 267.1174 & 267.1168 & 1.98 & $\mathrm{C}_{21} \mathrm{H}_{15}$ & - & + & - \\
\hline 269.1328 & 269.1325 & 1.10 & $\mathrm{C}_{21} \mathrm{H}_{17}$ & + & + & - \\
\hline 271.1485 & 271.1481 & 1.20 & $\mathrm{C}_{21} \mathrm{H}_{19}$ & + & + & - \\
\hline 273.1641 & 273.1638 & 1.06 & $\mathrm{C}_{21} \mathrm{H}_{21}$ & + & + & - \\
\hline 275.1796 & 275.1794 & 0.56 & $\mathrm{C}_{21} \mathrm{H}_{23}$ & + & + & - \\
\hline 281.1328 & 281.1325 & 1.08 & $\mathrm{C}_{22} \mathrm{H}_{17}$ & + & - & - \\
\hline 283.1485 & 283.1481 & 1.19 & $\mathrm{C}_{22} \mathrm{H}_{19}$ & + & + & - \\
\hline 285.1642 & 285.1638 & 1.45 & $\mathrm{C}_{22} \mathrm{H}_{21}$ & + & + & - \\
\hline 287.1798 & 287.1794 & 1.15 & $\mathrm{C}_{22} \mathrm{H}_{23}$ & + & + & - \\
\hline 289.1954 & 289.1951 & 1.00 & $\mathrm{C}_{22} \mathrm{H}_{25}$ & + & + & - \\
\hline 293.1330 & 293.1325 & 1.70 & $\mathrm{C}_{23} \mathrm{H}_{17}$ & - & + & - \\
\hline 295.1484 & 295.1481 & 0.93 & $\mathrm{C}_{23} \mathrm{H}_{19}$ & + & + & - \\
\hline 297.1642 & 297.1638 & 1.43 & $\mathrm{C}_{23} \mathrm{H}_{21}$ & - & + & - \\
\hline 299.1798 & 299.1794 & 1.12 & $\mathrm{C}_{23} \mathrm{H}_{23}$ & + & + & - \\
\hline 301.1955 & 301.1951 & 1.27 & $\mathrm{C}_{23} \mathrm{H}_{25}$ & - & + & - \\
\hline 303.2112 & 303.2107 & 1.63 & $\mathrm{C}_{23} \mathrm{H}_{27}$ & - & + & - \\
\hline 305.1324 & 305.1325 & -0.11 & $\mathrm{C}_{24} \mathrm{H}_{17}$ & + & - & - \\
\hline 307.1480 & 307.1481 & -0.36 & $\mathrm{C}_{24} \mathrm{H}_{19}$ & - & + & - \\
\hline 309.1638 & 309.1638 & -0.07 & $\mathrm{C}_{24} \mathrm{H}_{21}$ & + & + & - \\
\hline 311.1794 & 311.1794 & -0.05 & $\mathrm{C}_{24} \mathrm{H}_{23}$ & + & + & - \\
\hline 313.1951 & 313.1951 & -0.07 & $\mathrm{C}_{24} \mathrm{H}_{25}$ & + & + & - \\
\hline 315.2106 & 315.2107 & -0.45 & $\mathrm{C}_{24} \mathrm{H}_{27}$ & + & + & - \\
\hline 317.2264 & 317.2264 & 0.14 & $\mathrm{C}_{24} \mathrm{H}_{29}$ & - & + & - \\
\hline 319.2421 & 319.2420 & 0.15 & $\mathrm{C}_{24} \mathrm{H}_{31}$ & + & + & - \\
\hline
\end{tabular}

Notes. Summary of accurate mass measurements for the residue made from benzene irradiated at $19 \mathrm{~K}$ by $0.8 \mathrm{MeV}$ protons. Molecular formulae correspond to protonated molecules. Sections highlighted in italics represent formulae that correspond to polyphenyls (e.g., biphenyl, terphenyl, and quaterphenyl) and partially hydrogenated polyphenyls. Additionally, the presence $(+)$ or absence $(-)$ of these compounds in three different meteorites (two carbonaceous chondrites and one martian SNC meteorite) are also noted. A signal-to-noise ratio greater than three and a relative mass error of $<5 \mathrm{ppm}$ were used to define a "+" designation in meteorites, although signal-to-noise ratios of $10-100$, or higher, usually were observed. The relative mass error, in ppm, is calculated as $10^{6} \times\left(\right.$ mass $_{\text {experimental }}-$ mass $\left._{\text {theoretical }}\right) /\left(\right.$ mass $\left._{\text {theoretical }}\right)$. 
their formulas as assigned by accurate mass measurement, and the associated relative mass error.

An important purpose of our work was to compare the products of proton-irradiated benzene with organics found in meteorites. To do this, we extracted samples from Murchison (CM2 carbonaceous chondrite) and Orgueil (CI1 carbonaceous chondrite) powders in a method identical to that described above for the analysis of the benzene residue. Of the 69 diagnostic ions listed in Table 1 for the fingerprint of irradiated benzene ice, we found that Murchison showed 60 of them and Orgueil showed 67. Masses corresponding to biphenyl, terphenyl, quaterphenyl, and their partially hydrogenated derivatives were found in both meteorites. Both biphenyl and terphenyl were reported previously in carbonaceous chondrites (Naraoka et al., 2000; Pizzarello et al., 2008). This strong overlap of organic compounds is suggestive of, although not conclusive evidence for, a connection between the radiation chemistry of frozen benzene and the numerous aromatic compounds observed in carbonaceous chondrites. Fig. 4 shows the APPI mass spectrum of the Murchison meteorite dichloromethane extract along with close-up regions of selected mass ranges (where protonated terphenyls appear). The benzene residue masses that are detected in Murchison (as well as Orgueil) are all low intensity peaks, which suggest that these compounds are likely low abundance products.

To probe the extent of our conclusions, we also analyzed a sample from RBT 04262 - a martian shergottite. RBT 04262 has undergone high temperature igneous processing rather than aqueous alteration (Usui et al., 2010). Only one of the 69 diagnostic ions of Table 1 was present in this martian meteorite, and it is unknown whether this ion is from either the same molecule(s) or (a) structural isomer(s) measured in the benzene residue. Nonetheless, these results suggest that these organics are not all shared by extraterrestrial materials and that the synthesis and preservation of these organics may require an environment such as icy organic mantles of interstellar grains that eventually get incorporated into the meteorite parent body.

\section{Discussion}

\subsection{Radiation chemistry of benzene}

The analyses shown in Figs. 2 and 3 provide compositional "snapshots" of the organics made from $\mathrm{C}_{6} \mathrm{H}_{6}$ at the radiation dose employed. Although only one such dose (average energy: $119 \mathrm{eV}$ molecule $\mathrm{e}^{-1}$ ) was used for the samples that were analyzed, it can be considered a fair compromise between the much smaller radiation doses expected for the middle of dense interstellar clouds and the much larger ones for diffuse interstellar clouds. In any case, the IR spectra of irradiated benzene showed little, if any, changes as our final dose was reached, suggesting that an equilibrium state had been achieved. Smaller doses might give somewhat less product, but larger ones are not expected to give more.

The formation of polyphenyls from benzene, which we report here for the solid state, has been known for over 50 years for liquid- and gas-phase experiments. Such syntheses have been conducted with a variety of energy sources $\left({ }^{1} \mathrm{H}\right.$, ${ }^{4} \mathrm{He}$, and ${ }^{12} \mathrm{C}$ ions, $\gamma$ rays, radio frequency (spark) discharge), but not in the solid phase nor at the low temperatures used here (Gordon et al., 1958; LaVerne and Araos, 2002; Lee et al., 2007a,b).

Beyond our identification of polyphenyls and other products from irradiated benzene ice, one of our more striking conclusions is that there is no evidence for significant yields of PAHs in any of our experiments. As before, Fig. 1 illustrates that polyphenyls are composed of benzene rings (phenyl groups) linked only by $\mathrm{C}-$ $\mathrm{C}$ bonds, whereas PAHs are made of rings that are fused (Fig. 1, naphthalene for example). Our results suggest that PAHs in meteorites are not constructed by radiation processes involving smaller rings, but perhaps by the accumulation of atoms or molecular fragments. Once rings are formed; however, it appears to be relatively easy to make more complex molecules.

In astrophysical environments, PAHs have not been identified in interstellar ices; however, significant hydrogenation of PAHs in interstellar ices may preclude their spectroscopic detection. Therefore, a low temperature synthesis for PAHs cannot be ruled out. For

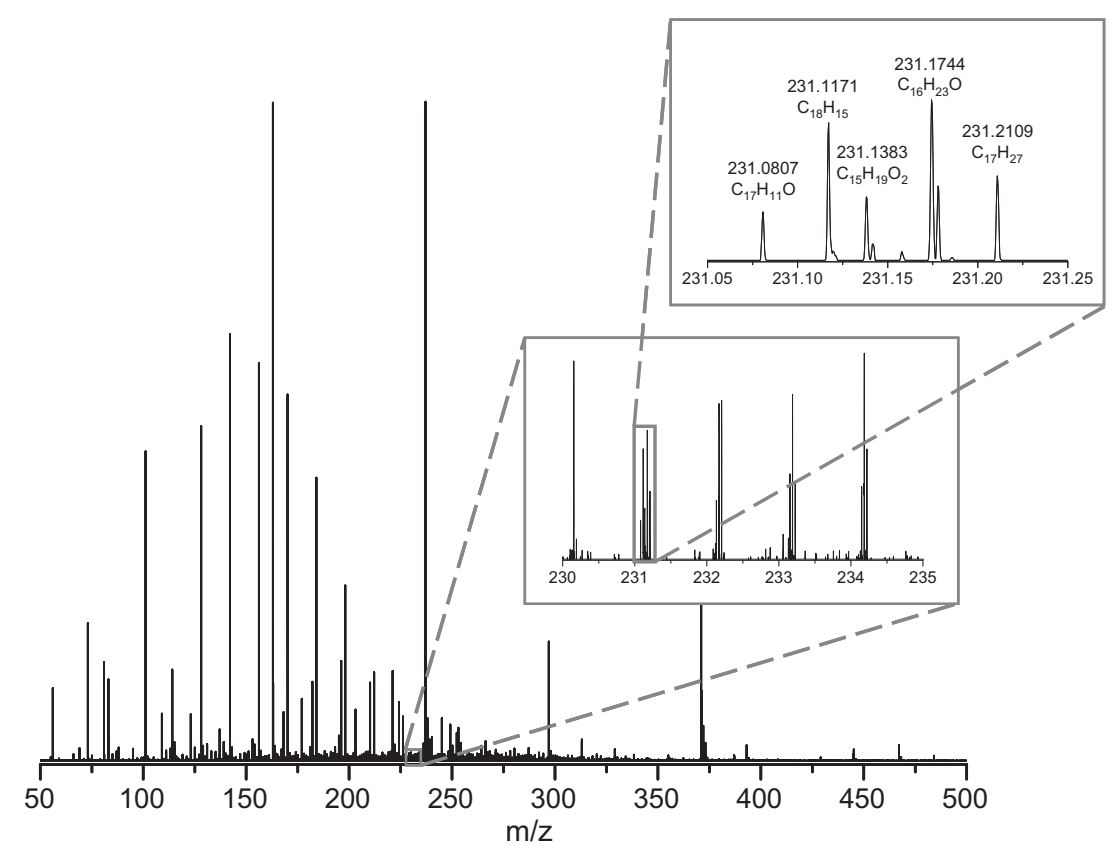

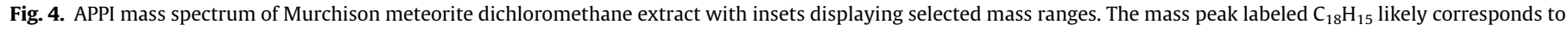
protonated terphenyl. 
example, experiments (along with electronic structure calculations) have shown that naphthalene (the simplest PAH) can be synthesized from vinylacetylene and phenyl radical in a low temperature environment (Parker et al., 2012), the latter precursor molecule produced possibly from irradiation and/or photolysis of benzene. Thus, benzene may still play a role in the synthesis of PAHs if other reagents are present.

The published results on chemical products from irradiated benzene stand in sharp contrast to the mechanistic details that are available - an undesirable situation. As a $\mathrm{MeV}$ ion passes through an ice it will produce a chain of ionizations and excitations that effect most of the resulting chemistry, with the dominant agent of change being secondary electrons with energies below $100 \mathrm{eV}$. An expected result with benzene will be the formation of phenyl radicals ( $\left.{ }^{\circ} \mathrm{C}_{6} \mathrm{H}_{5}\right)$ either through single step $\mathrm{C}-\mathrm{H}$ bond breakage or a multi-step process perhaps involving solid phase ion-molecule reactions. In either case, dimerization of the resulting ${ }^{\circ} \mathrm{C}_{6} \mathrm{H}_{5}$ radicals will produce $\left(\mathrm{C}_{6} \mathrm{H}_{5}\right)_{2}$, the biphenyl that we and others have observed. Dimerization is also a key step in the formation of other astronomical molecules, such as ethane $\left(\mathrm{C}_{2} \mathrm{H}_{6}\right)$ (Moore and Hudson, 1998), hydrogen peroxide $\left(\mathrm{H}_{2} \mathrm{O}_{2}\right)$ (Moore and Hudson, 2000), and ethylene glycol $\left(\mathrm{C}_{2} \mathrm{H}_{6} \mathrm{O}_{2}\right)$ (Hudson and Moore, 2000). We note, however, that this straightforward formation mechanism has been shown to be incomplete through work by LaVerne and colleagues (LaVerne and Araos, 2002). When radical scavengers are added to benzene before irradiation, to react with the $\mathrm{C}_{6} \mathrm{H}_{5}$ radicals produced, some biphenyl formation is still observed. Excited states of benzene or the benzene radical cation may play a role, but in both cases details remain to be determined (Baidak et al., 2011).

\subsection{Comparisons to meteorite chemistry}

We have compared our benzene residue "fingerprint" results to the APPI mass spectra of different meteorite extracts to investigate possible similarities to the radiation chemistry of frozen benzene and to determine if this type of cold, radiation induced aromatic chemistry may have influenced meteoritic and hence early Solar System chemistry. Table 1 shows that there are compositional similarities between the mass spectra of our benzene residue and the meteorites, but important differences exist. Fig. 5 compares our Orgueil meteorite and benzene residue results for three separate mass regions, and clearly demonstrates that the mass spectrum for Orgueil is more complex than that of the residue. However, due to the broad ionization of APPI and the elemental complexity, the peaks in the meteorite spectra that are not matched by those in the residue's spectra likely correspond to compounds other than aromatic hydrocarbons. At the measured relative mass error
$(<2 \mathrm{ppm})$ these other peaks are likely due to molecules containing nitrogen, oxygen, or both.

Furthermore, higher molecular weight products detected in the benzene residue were not observed in meteorite extracts, for which we can offer four possible explanations. First, larger molecules are produced more readily in the pure benzene ice than in the dilute ices likely involved in the formation of the meteorites. Second, benzene in astrophysical ices would likely be a minor component (in both absolute and relative abundances compared with $\mathrm{H}_{2} \mathrm{O}, \mathrm{CO}, \mathrm{CO}_{2}, \mathrm{CH}_{3} \mathrm{OH}$, etc.), and it would probably react with other species rather than itself to form polyphenyls. Third, the extraction method we employed is less effective for our meteorites than for our benzene residue. Finally, the abundances of these compounds in the meteorites decrease as the molecular weight increases to the point of being undetectable in our analysis. In any case, our results demonstrate that the irradiation of a single molecule, benzene, can lead to the production of numerous complex organic molecules and that much of their mass spectral signature is shared by at least two carbonaceous chondrites.

These types of experiments can be extended to other aromatic molecules such as nitrogen heterocycles (e.g., pyridine, pyrimidine, purine, and triazine). For instance, the nucleobases uracil and cytosine have been synthesized from pyrimidine-water ices under low temperature conditions and UV photolysis (Nuevo et al., 2009, 2012). We speculate that similar syntheses of nucleobases (and other complex products) can also occur via proton irradiation (cosmic rays) of pyrimidine-water ices. Similar comparisons using high-resolution mass spectral fingerprinting of these mixedmolecular ice residues and meteorite extracts would also be possible.

\subsection{Potential relevance to Titan}

We also carried out proton irradiation experiments of benzene at $150 \mathrm{~K}$ and analyzed the residue by APPI-MS. The APPI mass spectra for the residues of the $19 \mathrm{~K}$ and $150 \mathrm{~K}$ experiments were identical (results not shown). A portion of this temperature range (130$150 \mathrm{~K}$ ) overlaps relevant Titan temperatures where benzene was detected by the Cassini spacecraft (Waite et al., 2007). Although benzene has been detected only in the gas phase (the Cassini/Huygens instrumentation was far more sensitive to gases), the presence of condensed benzene remains likely, since benzene in Titan's upper atmosphere is close to its saturation vapor pressure (Waite et al., 2007). Furthermore, Cassini measurements of Titan's upper atmosphere revealed evidence of heavy positively charged (100-350 Da) and negatively charged (20-8000 Da) ions (Waite et al., 2007). Thus, biphenyl may be a feasible candidate in Titan's atmosphere as well as larger molecular weight polyphenyls,

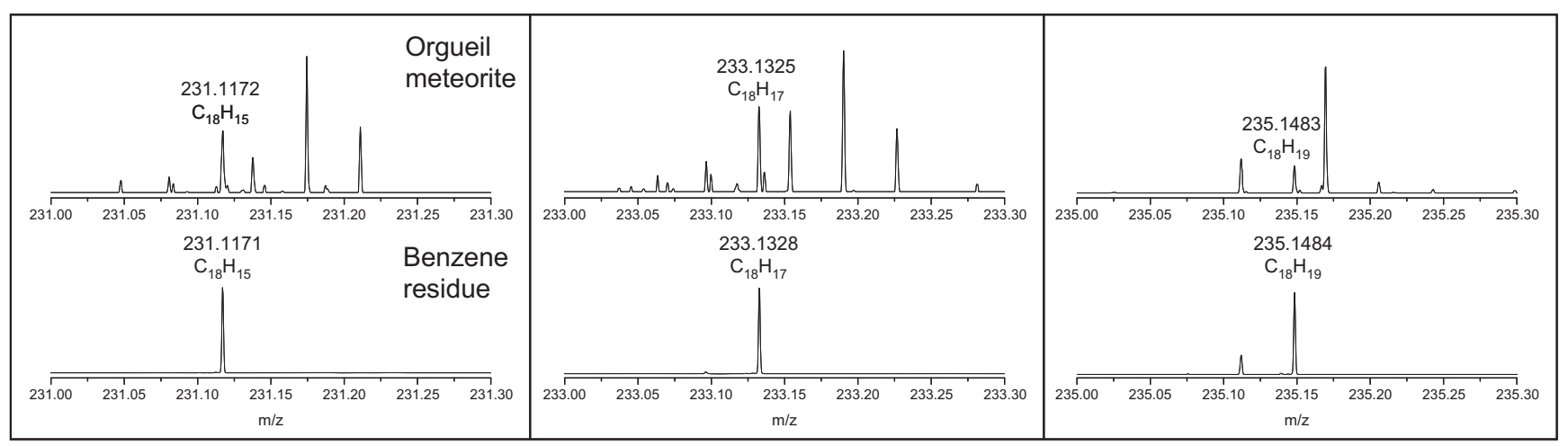

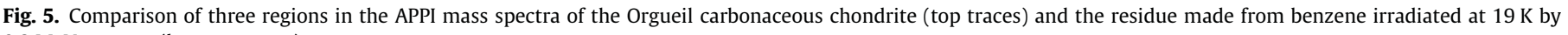
$0.8 \mathrm{MeV}$ protons (bottom traces). 
especially if they can accumulate into aerosol particles. Furthermore, polyphenyls (biphenyl and terphenyls) are known to have good radiation resistance and thermal stability (Delitsky and McKay, 2010), which would aid their survival in Titan's atmosphere. However, additional experiments that simulate more realistic Titan conditions (e.g., EUV radiation, benzene-nitrogenmethane mixtures, benzene-tholin mixtures, and gas phase reactions) are required to establish if polyphenyls are good candidates to account for the high molecular masses detected in Titan's atmosphere.

\section{Summary and conclusions}

By means of GC-MS and APPI-FTMS, we measured a highly complex suite of organic compounds in the residue made from benzene irradiated at $19 \mathrm{~K}$ by $0.8 \mathrm{MeV}$ protons. Our analysis unambiguously identified numerous polyphenyls and also suggested the presence of partially hydrogenated polyphenyls and other complex aromatic compounds. The strong overlap of low molecular weight organic compounds suggests a connection between the radiation chemistry of benzene and the numerous aromatic compounds observed in (at least two) carbonaceous meteorites. Furthermore, radiation-induced reactions provide a facile mechanism for the extensive hydrogenation (likely) observed in meteoritic organics. Our results concur with a low temperature origin for meteoritic aromatic compounds, most likely on icy organic mantles of interstellar grains. Alternative routes for the synthesis of the organics involving the meteorite parent body, such as synthesis during periods of aqueous alteration, may be difficult because benzene is relatively non-reactive, the formation of new $\mathrm{C}-\mathrm{C}$ bonds have high energetic barriers, and cosmic rays will have limited penetration depth. On the other hand, gamma radiation produced from the decay of radionuclides within the meteorite parent body over an extended timescale may have facilitated similar chemical reactions possibly though radiation-induced "circle" reactions (Sephton and Gilmour, 2000) involving simultaneous bond synthesis and breaking. This possibility may explain why Murchison and Orgueil meteorites had similar profiles of low molecular weight hydrocarbons despite experiencing different degrees of aqueous alteration.

Finally, applications of the radiation chemistry of low temperature benzene may extend beyond asteroids and meteoritics. The radiation chemistry (and photochemistry) of benzene in Titan's atmosphere is likely to produce complex products, and so polyphenyls also may be present in Titan's atmosphere and precursors to larger haze particles. Interstellar applications of low temperature polyphenyl synthesis from benzene also are possible.

\section{Acknowledgments}

The authors thank Marla Moore (NASA GSFC), Jason Dworkin (NASA GSFC), Jim Cleaves (Institute for Advanced Study), and an anonymous reviewer for helpful suggestions regarding this research or manuscript. We also thank Cecilia Satterwhite (NASA JSC), Kevin Righter (NASA JSC), and the Meteorite Working Group for providing the RBT 04262 meteorite sample, Linda Welzenbach and Tim McCoy (Smithsonian National Museum of Natural History) for providing the Murchison meteorite sample, Pascale Ehrenfreund (The George Washington University) for providing the Orgueil meteorite sample, and Andrew Mattioda (NASA Ames) for providing some of the PAH standards used in this study. In addition, we thank Steve Brown, Tom Ward, and Eugene Gerashchenko (Radiation Effects Facility at NASA GSFC) for operation of the proton accelerator. M.P.C. was partially supported by a NASA Postdoctoral Program fellowship administered by Oak Ridge Associated Universities through a contract with NASA. This research was supported by the NASA Astrobiology Institute and the Goddard Center of Astrobiology.

\section{References}

Baidak, A., Badali, M., LaVerne, J.A., 2011. Role of the low-energy excited states in the radiolysis of aromatic liquids. J. Phys. Chem. A 115, 7418-7427.

Bernstein, M.P., Sandford, S.A., Allamandola, L.J., Gillette, J.S., Clemett, S.J., Zare, R.N., 1999. UV irradiation of polycyclic aromatic hydrocarbons in ices: Production of alcohols, quinones, and ethers. Science 283, 1135-1138.

Bernstein, M.P., Elsila, J.E., Dworkin, J.P., Sandford, S.A., Allamandola, L.J., Zare, R.N. 2002. Side group addition to the polycyclic aromatic hydrocarbon coronene by ultraviolet photolysis in cosmic ice analogs. Astrophys. J. 576, 1115-1120.

Callahan, M.P. et al., 2011. Carbonaceous meteorites contain a wide range of extraterrestrial nucleobases. Proc. Nat. Acad. Sci. USA 108, 13995-13998.

Cernicharo, J. et al., 2001. Infrared Space Observatory's discovery of C-4H2, C-6H2, and benzene in CRL 618. Astrophys. J. 546, L123-L126.

Chyba, C., Sagan, C., 1992. Endogenous production, exogenous delivery and impactshock synthesis of organic-molecules - An inventory for the origins of life. Nature 355, 125-132.

Ciesla, F.J., Sandford, S.A., 2012. Organic synthesis via irradiation and warming of ice grains in the solar nebula. Science 336, 452-454.

Cooper, G., Kimmich, N., Belisle, W., Sarinana, J., Brabham, K., Garrel, L., 2001. Carbonaceous meteorites as a source of sugar-related organic compounds for the early Earth. Nature 414, 879-883.

Cooper, G., Reed, C., Nguyen, D., Carter, M., Wang, Y., 2011. Detection and formation scenario of citric acid, pyruvic acid, and other possible metabolism precursors in carbonaceous meteorites. Proc. Nat. Acad. Sci. USA 108, 14015-14020.

Coustenis, A. et al., 2003. Titan's atmosphere from ISO mid-infrared spectroscopy. Icarus 161, 383-403.

Cronin, J.R., Pizzarello, S., 1997. Enantiomeric excesses in meteoritic amino acids. Science 275, 951-955.

Delitsky, M.L., McKay, C.P., 2010. The photochemical products of benzene in Titan's upper atmosphere. Icarus 207, 477-484.

Flasar, F.M. et al., 2005. Titan's atmospheric temperatures, winds, and composition. Science 308, 975-978.

Frenklach, M., Feigelson, E.D., 1989. Formation of polycyclic aromatic-hydrocarbons in circumstellar envelopes. Astrophys. J. 341, 372-384.

Gordon, S., Vandyken, A.R., Doumani, T.F., 1958. Identification of products in the radiolysis of liquid benzene. J. Phys. Chem. 62, 20-24.

Gudipati, M. S., Yang, R., 2012. In-situ probing of radiation-induced processing of organics in astrophysical ice analogs-novel laser desorption laser ionization time-of-flight mass spectroscopic studies. The Astrophysical Journal Letters, 756: L24 (5 pp).

Hanold, K.A., Fischer, S.M., Cormia, P.H., Miller, C.E., Syage, J.A., 2004. Atmospheric

pressure photoionization. 1. General properties for LC/MS. Anal. Chem. 76, $2842-2851$.

Hockaday, W.C Grannas, A.M. Kim, S, Hatcher, P.G 2006, Direct molecular evidence for the degradation and mobility of black carbon in soils from ultrahigh-resolution mass spectral analysis of dissolved organic matter from a fire-impacted forest soil. Organic Geochem. 37, 501-510.

Hudson, R.L., Moore, M.H., 2000. IR spectra of irradiated cometary ice analogues containing methanol: A new assignment, a reassignment, and a nonassignment. Icarus 145, 661-663.

Hudson, R.L., Moore, M.H., 2003. Solid-phase formation of interstellar vinyl alcohol. Astrophys. J. 586, L107-L110.

Hudson, R.L., Moore, M.H., 2004. Reactions of nitriles in ice's relevant to Titan, comets, and the interstellar medium: Formation of cyanate ion, ketenimines, and isonitriles. Icarus 172, 466-478.

Kerridge, J.F., Chang, S., Shipp, R., 1987. Isotopic characterization of kerogen-like material in the Murchison carbonaceous chondrite. Geochim. Cosmochim. Acta 51, 2527-2540.

Kvenvolden, K. et al., 1970. Evidence for extraterrestrial amino-acids and hydrocarbons in Murchison meteorite. Nature 228, 923-926.

LaVerne, J.A., Araos, M.S., 2002. Heavy ion radiolysis of liquid benzene. J. Phys. Chem. A 106, 11408-11413.

Lee, S., Chen, H.F., Chin, C.J., 2007a. Spectroscopic study of carbonaceous dust particles grown in benzene plasma. J. Appl. Phys. 101, 113303-1-113303-8.

Lee, S., Chen, H.F., Peng, J.W., 2007b. Investigation of soot deposition and composition in a radio frequency plasma of benzene. Plasma Chem. Plasma Process. 27, 256-266.

Meierhenrich, U.J., Caro, G.M.M., Bredehoft, J.H., Jessberger, E.K., Thiemann, W.H.P., 2004. Identification of diamino acids in the Murchison meteorite. Proc. Nat. Acad. Sci. USA 101, 9182-9186.

Moore, M.H., Hudson, R.L., 1998. Infrared study of ion-irradiated water-ice mixtures with hydrocarbons relevant to comets. Icarus $135,518-527$.

Moore, M.H., Hudson, R.L., 2000. IR detection of $\mathrm{H}_{2} \mathrm{O}_{2}$ at $80 \mathrm{~K}$ in ion-irradiated laboratory ices relevant to Europa. Icarus 145, 282-288.

Mullie, F., Reisse, J., 1987. Organic-matter in carbonaceous chondrites. Topics Curr. Chem. 139, 83-117.

Naraoka, H., Shimoyama, A., Harada, K., 2000. Isotopic evidence from an Antarctic carbonaceous chondrite for two reaction pathways of extraterrestrial PAH formation. Earth Planet. Sci. Lett. 184, 1-7. 
Nuevo, M., Milam, S.N., Sandford, S.A., Elsila, J.E., Dworkin, J.P., 2009. Formation of uracil from the ultraviolet photo-irradiation of pyrimidine in pure $\mathrm{H}_{2} \mathrm{O}$ ices Astrobiology 9, 683-695.

Nuevo, M., Milam, S.N., Sandford, S.A., 2012. Nucleobases and prebiotic molecules in organic residues produced from the ultraviolet photo-irradiation of pyrimidine in $\mathrm{NH}_{3}$ and $\mathrm{H}_{2} \mathrm{O}+\mathrm{NH}_{3}$ ices. Astrobiology 12, 295-314.

Oró, J., 1961. Comets and formation of biochemical compounds on primitive Earth. Nature 190, 389-390.

Parker, D.S.N. et al., 2012. Low temperature formation of naphthalene and its role in the synthesis of PAHs (Polycyclic Aromatic Hydrocarbons) in the interstella medium. Proc. Nat. Acad. Sci. USA 109, 53-58.

Pizzarello, S., Huang, Y., Alexandre, M.R., 2008. Molecular asymmetry in extraterrestrial chemistry: Insights from a pristine meteorite. Proc. Nat. Acad. Sci. USA 105, 3700-3704.

Robb, D.B., Covey, T.R., Bruins, A.P., 2000. Atmospheric pressure photoionisation: An ionization method for liquid chromatography-mass spectrometry. Anal. Chem. 72, 3653-3659.

Ruiterkamp, R., Peeters, Z., Moore, M.H., Hudson, R.L., Ehrenfreund, P., 2005. A quantitative study of proton irradiation and UV photolysis of benzene in interstellar environments. Astron. Astrophys. 440, 391-402.

Schmitt-Kopplin, P. et al., 2010. High molecular diversity of extraterrestrial organic matter in Murchison meteorite revealed 40 years after its fall. Proc. Nat. Acad. Sci. USA 107, 2763-2768.

Sephton, M.A., 2002. Organic compounds in carbonaceous meteorites. Nat. Product Rep. 19, 292-311.

Sephton, M.A., Gilmour, I., 2000. Aromatic moieties in meteorites: Relics of interstellar grain processes? Astrophys. J. 540, 588-591.
Sephton, M.A., Pillinger, C.T., Gilmour, I., 1998. Delta(13)C of free and macromolecular aromatic structures in the Murchison meteorite. Geochim. Cosmochim. Acta 62, 1821-1828.

Sephton, M.A., Pillinger, C.T., Gilmour, I., 2000. Aromatic moieties in meteoritic macromolecular materials: Analyses by hydrous pyrolysis and delta(13)C of individual compounds. Geochim. Cosmochim. Acta 64, 321-328.

Short, L.C., Cai, S.S., Syage, J.A., 2007. APPI-MS: Effects of mobile phases and VUV lamps on the detection of PAH compounds. J. Am. Soc. Mass Spectromet. 18 589-599.

Strazzulla, G., Baratta, G.A., 1991. Laboratory study of the IR-spectrum of ionirradiated frozen benzene. Astron. Astrophys. 241, 310-316.

Thrower, J.D. et al., 2012. Experimental evidence for the formation of highly superhydrogenated polycyclic aromatic hydrocarbons through $\mathrm{H}$ atom addition and their catalytic role in H-2 formation. Astrophys. J., 752: 3 (6 pp).

Usui, T., Sanborn, M., Wadhwa, M., McSween, H.Y., 2010. Petrology and trace element geochemistry of Robert Massif 04261 and 04262 meteorites, the firs examples of geochemically enriched lherzolitic shergottites. Geochim. Cosmochim. Acta 74, 7283-7306.

Waite, J.H. et al., 2005. Ion neutral mass spectrometer results from the first flyby of Titan. Science 308, 982-986.

Waite, J.H. et al., 2007. The process of tholin formation in Titan's upper atmosphere. Science 316, 870-875.

Yuen, G.U., Kvenvolden, K.A., 1973. Monocarboxylic acids in Murray and Murchison carbonaceous meteorites. Nature 246, 301-302. 